\title{
机械结构冲击载荷稀疏识别方法研究*
}

\author{
乔百杰 1,2 陈雪峰 1,2 刘金金金 1,2 王诗涁 1,2 \\ (1. 西安交通大学机械工程学院 西安 710049;
}

2. 西安交通大学机械制造系统工程国家重点实验室 西安 710049)

\begin{abstract}
摘要: 冲击载荷识别在结构健康监测、动力学优化设计、铣削力测量等领域扮演重要角色。然而, 现有的基于 L2 范数的冲 击载荷识别正则化方法在识别精度、稳定性、计算效率、参数选取等方面均存在瓶颈和局限。近年来兴起的稀疏正则化理论 为冲击载荷识别提供了一种新的探索途径。充分利用冲击载荷在时域内稀疏的先验信息, 提出冲击载荷稀疏识别新方法, 通 过最小化 L1 罚函数项取代传统的最小化 L2 罚函数项, 建立基于 L1 范数的稀疏识别正则化模型, 突破基于 L2 范数的冲击 载荷识别方法精度低的瓶颈。基于 L1 范数的稀疏识别方法与基于 L2 范数的 Tikhonov 正则化方法在机械结构单源和多源冲 击载荷识别中进行了对比。薄板结构冲击载荷识别试验表明：基于 L1 范数的正则化解在时域内非常稀疏, 冲击载荷非加载 区噪声被极大地抑制; 稀疏识别方法在重构冲击载荷时间历程、稳定性和计算效率方面均优于传统的 Tikhonov 方法。
\end{abstract}

关键词: 冲击载荷识别; 稀疏识别; 正则化; 反问题

中图分类号: TB123

\section{Sparse Identification of Impact Force Acting on Mechanical Structures}

\author{
QIAO Baijie $^{1,2}$ CHEN Xuefeng ${ }^{1,2}$ LIU Jinxin ${ }^{1,2}$ WANG Shibin ${ }^{1,2}$
}

(1. School of Mechanical Engineering, Xi’an Jiaotong University, Xi’an 710049;

2. The State Key Laboratory for Manufacturing Systems Engineering, Xi'an Jiaotong University, Xi'an 710049)

\begin{abstract}
Impact force identification plays an important role in structural health monitoring, dynamics optimization design, milling force measurement, etc. However, the traditional L2 norm-based regularization methods run into the bottleneck and limitation on identification accuracy, stability, computational efficiency, parameter selection, etc. Sparse regularization theory widely developed in recent years is a promising technique for impact force identification. Considering the prior information that the impact force is sparse in time domain, a general impact force sparse identification model is proposed, where the traditional minimization L2 norm penalty is replaced by the minimization L1 norm penalty. A sparse regularization model based on L1 norm is established, leading to breaking through the limitation of the low identification accuracy of the traditional L2 norm-based regularization methods in impact force identification. The sparse identification method based on L1 norm is examined and compared with the Tikhonov regularization method based on L2 norm under single impact force and multiple impact forces. Experimental results of identifying impact force acting on a thin plate structure demonstrate that the sparse regularization solution based on L1 norm is sufficiently sparse in time domain, where the noise in the unloading stage of impact force is greatly inhibited; the proposed sparse identification method has great advantages of reconstructing impact force history, stability and computational efficiency over the traditional Tikhonov regularization method.
\end{abstract}

Key words: impact force identification; sparse identification; regularization; inverse problem

\section{0 前言}

航空发动机风扇叶片、风力发电机叶片、机翼 等机械结构, 工作环境恶劣, 在服役和维护过程中 不可避免地遭受到冰雨、飞鸟、维修工具等外来物

* 国家自然科学基金(51705397)、中国博士后基金(2017M610636)和国家重 点基础研究发展计划(2015CB057400)资助项目。20180409 收到初稿, 20181019 收到修改稿
的冲击。冲击损伤累积下来会对机械结构的完整性 以及承载能力造成隐患，从而造成巨大的经济损失 或人员伤亡。这使得机械结构冲击载荷识别的研究 受到极大关注。载荷识别是已知系统动态特性和 实测响应, 反演重构作用于机械结构的未知外来 载荷 ${ }^{[1-3]}$ 。不同于动力响应求解正问题, 载荷识别反 问题是典型的病态或者不适定性问题, 即不满足 Hadmard 存在性、唯一性和稳定性三准则, 其病态 特性意味着冲击响应中的微弱噪声就会导致解的巨 
大偏差 ${ }^{[4]}$ 。因此, 利用经典的最小二乘法直接求解 是不可行的, 必须研究载荷识别的正则化方法, 通 过添加正则化约束条件, 将不适定性(Ill-posed)问题 变成适定性(Well-posed)问题。

经过几十年的发展, 结构动载荷识别正则化方 法主要发展为频域法和时域法 ${ }^{[5]}$ 。频域法利用系统 频响函数(Frequency response function, FRF)在频域 内求解未知载荷。频域载荷识别方法的研究较早, 理论也比较成熟。比如, 美国普渡大学 DOYLE ${ }^{[6]}$ 应用频域解卷积法由实测加速度信号估计冲击力频 谱, 进而通过快速傅里叶逆变换获得冲击载荷时间 历程。时域载荷识别方法不同于传统的频域法, 无 须在每一个频点对 FRF 矩阵求逆, 而是根据载荷与 系统传递函数的卷积关系, 通过解卷积获得载荷的 时域离散信号, 能够处理瞬态信号, 在冲击载荷识 别中应用较多。基于 L2 范数的 Tikhonov、截断奇 异值分解(TSVD)、函数逼近等正则化方法广泛应用 在时域载荷识别中 ${ }^{[6,7]}$ 。2003 年, JACQUELIN 等 ${ }^{[8]}$ 讨论了广义 SVD、Tikhonov 和 TSVD 三种方法在载 荷时域解卷积中的应用, 并通过识别作用于 Al-5054 铝板冲击力进行对比。2011 年, BOUKRIA 等 ${ }^{[9]}$ 利 用 Tikhonov 正则化方法和 $\mathrm{L}$ 曲线参数选择准则, 识 别了作用在圆形钢板的冲击载荷。2011 年, 湖南大 学 WANG 等 ${ }^{[7]}$ 基于 Tikhonov 提出了一个新型正则 化算子以识别作用在复合材料层合圆柱壳结构载 荷 $^{[7]}$ 。2016 年, 悉尼大学 KALHORI 等 ${ }^{[10]}$ 利用 PZT 压电传感器测量振动响应信号, 采用 Tikhonov 方法 实现了复合材料层合板冲击载荷识别。在函数逼近 法方面, 南京航天航空大学 YAN ${ }^{[11]}$ 利用两个 $1 / 4$ 周 期的正弦函数逼近作用于复合材料加筋板的冲击载 荷。2015 年以来, 作者团队 ${ }^{[12-14]}$ 围绕冲击载荷识别 的函数逼近法取得了一些研究成果。通过选择与冲 击载荷形貌匹配的基函数，提出了基于小波多分辨 分析的三次 $\mathrm{B}$ 样条尺度函数 ${ }^{[12]}$ 和基于三次 $\mathrm{B}$ 样条函 数配置 ${ }^{[13]}$ 的冲击载荷识别方法, 悬臂梁结构试验表 明提出的方法优于 TSVD 方法。然而, 上述基于 L2 范数的载荷识别方法(即遵循全局能量最小化原则) 对响应噪声、振动初始条件、正则化参数比较敏感, 识别精度达到瓶颈。

近 10 年,受新理论压缩感知(Compressed sensing) 的推动, 基于 $\mathrm{L} 1$ 范数的稀疏约束作为一个基本正 则化条件受到空前的关注, 并迅速成为信号、图像 处理及相关领域的前沿研究课题 ${ }^{[15-17]}$ 。基于 L1 范 数的正则化方法的解通常是稀疏的, 而通过控制待 求变量能量的 L2 范数正则化方法的解常常是非稀 疏的 ${ }^{[15]}$ 。稀疏理论的出现为冲击载荷识别提供了一
种全新思路。从稀疏的角度来说, 冲击载荷相对其 采样数据长度在时域内是非常稀疏的，仅仅在冲击 加载区附近有较大值, 而非加载区全部为零。本文 充分考虑冲击载荷的时域稀疏性, 构造冲击载荷识 别控制方程的稀疏约束条件，建立基于 L1 范数的 冲击载荷稀疏识别模型, 实现机械结构冲击载荷时 间历程的高保真重构, 并通过薄板结构的单源、多 源冲击载荷验证基于 L1 范数稀疏识别方法相对传 统 L2 范数正则化方法的优势。

\section{1 冲击载荷识别控制方程}

\section{1 单源冲击载荷识别控制方程}

针对单输入和单输出 (Single-input-singleoutput, SISO) 的线性非时变机械系统, 假设零初始 条件 $y(0)=0$ 和 $\dot{y}(0)=0$, 则系统响应 $y(t)$ 可由输入 激励 $f(t)$ 和系统单位脉冲响应函数 $h(t)$ 的卷积关系 描述:

$$
y(t)=h(t) \otimes f(t)=\int_{0}^{t} h(t-\tau) f(\tau) \mathrm{d} \tau
$$

式中, $\otimes$ 表示卷积运算; $\tau$ 表示时移因子, 且满足 $t \geqslant \tau ; y(t)$ 表示系统响应, 比如加速度、速度、位 移、应变等便于测量的物理量。可知式(1)具有一阶 线性 Volterra 积分方程的形式, 建立了机械系统动 载荷作用点与响应测点间的数学关系。

式(1)描述的是一个正问题, 已知系统激励和系 统传递函数求系统响应，便是由 “因” 求 “果”。而 载荷识别是通过测量得到的系统响应结合系统模型 反演获得输入激励, 便是由 “果” 求 “因”。响应求 解是激励和系统脉冲响应函数的卷积, 反之, 载荷 识别则是输出响应和系统脉冲响应函数的解卷积。 因此, 载荷识别也称为载荷解卷积。由于实际测量 的响应为离散数据, 需要对式(1)离散化处理。进一 步, 式(1)的解卷积问题可记作矩阵-矢量形式

$$
\left(\begin{array}{c}
y\left(t_{1}\right) \\
y\left(t_{2}\right) \\
\vdots \\
y\left(t_{n-1}\right) \\
y\left(t_{n}\right)
\end{array}\right)=
$$

$$
\Delta t\left(\begin{array}{ccccc}
h(\Delta t) & 0 & \cdots & 0 & 0 \\
h(2 \Delta t) & h(\Delta t) & \cdots & 0 & 0 \\
\vdots & \vdots & & \vdots & \vdots \\
h((n-1) \Delta t) & h((n-2) \Delta t) & \cdots & h(\Delta t) & 0 \\
h(n \Delta t) & h((n-1) \Delta t) & \cdots & h(2 \Delta t) & h(\Delta t)
\end{array}\right)\left(\begin{array}{c}
f\left(t_{1}\right) \\
f\left(t_{2}\right) \\
\vdots \\
f\left(t_{n-1}\right) \\
f\left(t_{n}\right)
\end{array}\right)
$$


从而, 式(2)可记作更紧凑的矩阵-矢量形式

$$
\boldsymbol{y}=\boldsymbol{H} \boldsymbol{f}
$$

式中, $\boldsymbol{f} \in \mathbf{R}^{n}$ 表示待识别冲击载荷矢量; $\boldsymbol{y} \in \mathbf{R}^{n}$ 表 示系统响应矢量; 系统传递矩阵 $\boldsymbol{H} \in \mathbf{R}^{n \times n}$ 是一个具 有 Toeplitz 结构的下三角矩阵, 表征系统的动态特 性, 与激励点和响应点位置有关。

\section{2 多源冲击载荷识别控制方程}

当线性系统受到多个冲击载荷同时作用时, 系 统响应是各振源响应的线性叠加, 其中单个冲击载 荷产生的结构响应仍然可以通过该载荷与相应脉冲 响应函数的卷积(见式(3))得到。因此, 多输入多输 出(Multiple-input multiple-output, MIMO)系统的控 制方程可表示为如下矩阵-矢量形式

$$
\left(\begin{array}{c}
\boldsymbol{y}_{1} \\
\boldsymbol{y}_{2} \\
\vdots \\
\boldsymbol{y}_{M}
\end{array}\right)=\left(\begin{array}{cccc}
\boldsymbol{H}_{11} & \boldsymbol{H}_{12} & \cdots & \boldsymbol{H}_{1 N} \\
\boldsymbol{H}_{21} & \boldsymbol{H}_{22} & \cdots & \boldsymbol{H}_{2 N} \\
\vdots & \vdots & & \vdots \\
\boldsymbol{H}_{M 1} & \boldsymbol{H}_{M 1} & \cdots & \boldsymbol{H}_{M N}
\end{array}\right)\left(\begin{array}{c}
\boldsymbol{f}_{1} \\
\boldsymbol{f}_{2} \\
\vdots \\
\boldsymbol{f}_{N}
\end{array}\right)
$$

式中, $N$ 表示激励源数目; $M$ 表示响应点数目, 也 就是传感器数目; 子矩阵 $\boldsymbol{H}_{i j}$ 表示响应点 $i$ 和激励点 $j$ 间的传递矩阵。式(4)左边子矢量 $\boldsymbol{f}_{j}$ 对应第 $j$ 个激 励载荷, 而右边的子矢量 $\boldsymbol{y}_{i}$ 表示第 $i$ 个传感器记录 的时域响应。

为了统一表达, 上式也可记作紧凑的矩阵-矢量 表达形式

$$
\boldsymbol{y}=\boldsymbol{H} \boldsymbol{f}
$$

式中, 新定义的传递矩阵 $\boldsymbol{H} \in \mathbf{R}^{n M \times n N}$ 是一个 Toeplitz 分块矩阵。在这种情况下, 未知载荷矢量 $\boldsymbol{f}$ 可看作 由 $N$ 个载荷矢量组成的多通道矢量; 振动响应 $\boldsymbol{y}$ 可 看作由 $M$ 个响应矢量组成的多通道矢量。多源载荷 识别问题根据激励源和响应点数目分为三类情形: 欠定系统、正定系统和欠定系统。对于欠定系统, 基于 L2 范数的正则化方法比如 Tikhonov、TSVD 均不可获得唯一解。

由于传递矩阵 $\boldsymbol{H}$ 通常是病态甚至秩亏, 且随着 反问题维数的增大, 病态程度变得更加严重, 从而 变得对响应 $\boldsymbol{y}$ 中的噪声极其敏感。由于测量噪声在 振动测试过程中难以避免, 一个含有测量噪声的载 荷识别模型如下式

$$
\boldsymbol{y}=\boldsymbol{H} \boldsymbol{f}+\boldsymbol{w}
$$

式中, 矢量 $\boldsymbol{w} \in \mathbb{R}^{n}$ 表示不可避免的测量噪声和误 差。传递矩阵 $\boldsymbol{H}$ 可通过解析法、有限元数值法以及 模态试验法获得。其中, 模态试验法适用于各类复 杂的机械结构, 较为成熟, 可操作性强。

\section{2 冲击载荷稀疏识别模型}

\section{1 基于 $\mathrm{L} 2$ 范数的正则化方法}

Tikhonov 方法是应用最广泛的基于 L2 范数 的正则化方法, 在正则化理论发展过程中占据着 重要地位。Tikhonov 正则化方法通过添加约束 条件使不适定问题变得适定, 其核心思想是在残 差范数 $\|\boldsymbol{H} \boldsymbol{f}-\boldsymbol{y}\|_{2}$ 和解范数 $\|\boldsymbol{f}\|_{2}$ 之间寻找一个称 作正则化参数的加权因子 $\lambda$, 进而修正奇异值以 提升解的稳定性。Tikhonov 正则化的目标函数 为 ${ }^{[4,14]}$

$$
\underset{f}{\operatorname{minimize}}\|\boldsymbol{f}\|_{2} \quad \text { s.t. } \boldsymbol{y}=\boldsymbol{H} \boldsymbol{f}
$$

考虑到响应数据中不可避免的含有测量噪声, 则式(7)可写成

$$
\underset{\boldsymbol{f}}{\operatorname{minimize}}\|\boldsymbol{f}\|_{2} \quad \text { s.t. }\|\boldsymbol{y}-\boldsymbol{H} \boldsymbol{f}\|_{2} \leqslant \delta
$$

式中, $\delta$ 表示噪声水平。进一步可以写作无约束的 等价形式

$$
\underset{f}{\operatorname{minimize}}\|\boldsymbol{H} \boldsymbol{f}-\boldsymbol{y}\|_{2}^{2}+\lambda\|\boldsymbol{f}\|_{2}^{2}
$$

式中, $\lambda$ 表示恒为正的 Tikhonov 正则化参数。式(9) 就是广泛应用到反问题求解中的 Tikhonov 正则化 模型。对目标函数(9)关于 $\boldsymbol{f}$ 求导可得

$$
\left(\boldsymbol{H}^{\mathrm{T}} \boldsymbol{H}+\lambda \boldsymbol{I}\right) \boldsymbol{f}=\boldsymbol{H}^{\mathrm{T}} \boldsymbol{y}
$$

显然, 对于任意确定的 $\lambda$, 基于 $\mathrm{L} 2$ 范数的 Tikhonov 正则化模型都有唯一解:

$$
\boldsymbol{f}=\left(\boldsymbol{H}^{\mathrm{T}} \boldsymbol{H}+\lambda \boldsymbol{I}\right)^{-1} \boldsymbol{H}^{\mathrm{T}} \boldsymbol{y}
$$

可知, Tikhonov 正则化方法的关键是确定参数 $\lambda$ 。目前广泛使用的正则化参数选择准则为交叉检 验准则和 L 曲线准则 ${ }^{[4,14]}$ 。

\section{2 基于 L1 范数的正则化方法}

一般说来, Tikhonov 正则化方法是全局优化算 法, 通过最小化响应残差和解的 L2 范数的加权之 和(见式(9)), 获得的正则化解在整个时间历程都是 非零的, 即识别结果是不稀疏的。同时, 由于基于 L2 范数的 Tikhonov 正则化方法涉及到矩阵求逆, 当求解问题维度较大时, 计算效率较低 ${ }^{[17]}$ 。对于冲 击载荷而言, 其在时域内是非常稀疏的, 即仅仅在 冲击力的加载区是非零的, 非加载区的值全部为零。 因此, 可通过构造稀疏约束条件实现冲击载荷识别 反问题的正则化求解。

为了获得冲击载荷的稀疏解, 直观的处理方式 是最小化待识别冲击载荷矢量的非零元素数目

$$
\underset{\boldsymbol{f}}{\operatorname{minimize}}\|\boldsymbol{f}\|_{0} \quad \text { s.t. } \boldsymbol{y}=\boldsymbol{H} \boldsymbol{f}
$$


式中, $\|\boldsymbol{f}\|_{0}$ 表示冲击载荷矢量中非零元素的数目, 称为 $\mathrm{L} 0$ 范数。

基于 L0 范数的稀疏识别模型(12)中最小化 $\|\boldsymbol{f}\|_{0}$ 罚函数项取代了基于 L2 范数的 Tikhonov 模型(7)中 的最小化 $\|\boldsymbol{f}\|_{2}$ 罚函数项。然而, 基于 L0 范数的正 则化问题是一个 NP 难题, 直接对问题(12)求解是 非常困难的。CHEN 等 ${ }^{[18]}$ 用 L1 范数取代了模型(12) 中的 L0 范数, 将 NP 难题转化为一个可解的凸优 化问题。Donoho 证实了对于大多数反问题, 最小 化 L1 范数也可获得最优稀疏解 ${ }^{[19]}$ 。同样, 在冲击 载荷识别中, 用 L1 范数代替 L0 范数仍旧可以产 生稀疏解。这也就引出了基于 L1 范数的稀疏正则 化模型

$$
\underset{\boldsymbol{f}}{\operatorname{minimize}}\|\boldsymbol{f}\|_{1} \quad \text { s.t. } \boldsymbol{y}=\boldsymbol{H} \boldsymbol{f}
$$

式中, $\|\boldsymbol{f}\|_{1}=\sum_{i=1}^{n}\left|f_{i}\right|$ 表示冲击载荷矢量的 L1 范数,

即载荷矢量中所有元素的绝对值之和。与 L2 范数 正则化模型能量最小化准则不同, $\mathrm{L} 1$ 范数正则化模 型抑制了解的能量在时间尺度上的扩散, 保留了待 重构信号的稀疏特征。

此时, 基于 L1 范数的单源冲击载荷的稀疏识 别模型可由图 1 表示, 基于 L1 范数的多源冲击载 荷的稀疏识别模型可由图 2 表示。在两类稀疏识别 模型中, 响应矢量 $\boldsymbol{y}$ 如加速度冲击响应信号都是非 稀疏的。单源冲击载荷稀疏识别模型中的观测矩阵 $\boldsymbol{H}$ 是一个下三角矩阵, 待求冲击载荷矢量 $\boldsymbol{f}$ 仅有一 个(单源单次冲击)或者多个(单源多次冲击)非零区 间; 多源冲击载荷稀疏识别模型中的观测矩阵 $\boldsymbol{H}$ 是 一个下三角分块矩阵, 待求冲击载荷矢量 $\boldsymbol{f}$ 有多处 非零区间, 取决于源数目。

考虑到测量响应中常常伴随着不期望的测量误 差和环境噪声, 模型(13)的等式约束条件可转化为 不等式约束

$$
\underset{f}{\operatorname{minimize}}\|\boldsymbol{f}\|_{1} \quad \text { s.t. }\|\boldsymbol{y}-\boldsymbol{H} \boldsymbol{f}\|_{2}^{2} \leqslant \delta
$$

式中, 容差 $\delta$ 表示响应信号的噪声水平。对于基于 L1 范数的正则化, 一个等价于模型(14)的无约束凸 优化稀疏识别模型为

$$
\underset{f}{\operatorname{minimize}}\|\boldsymbol{H} \boldsymbol{f}-\boldsymbol{y}\|_{2}^{2}+\lambda\|\boldsymbol{f}\|_{1}
$$

式中, $\lambda$ 表示 $\mathrm{L} 1$ 范数的正则化参数, 其在残差 $\|\boldsymbol{H} \boldsymbol{f}-\boldsymbol{y}\|_{2}^{2}$ 和稀疏正则化算子 $\|\boldsymbol{f}\|_{1}$ 间建立了折中关 系。式(15)中的 L1 范数项 $\|\boldsymbol{f}\|_{1}$ 具有促使未知冲击载 荷中较小数值变成零的作用, 从而诱导得到冲击载 荷的稀疏解。

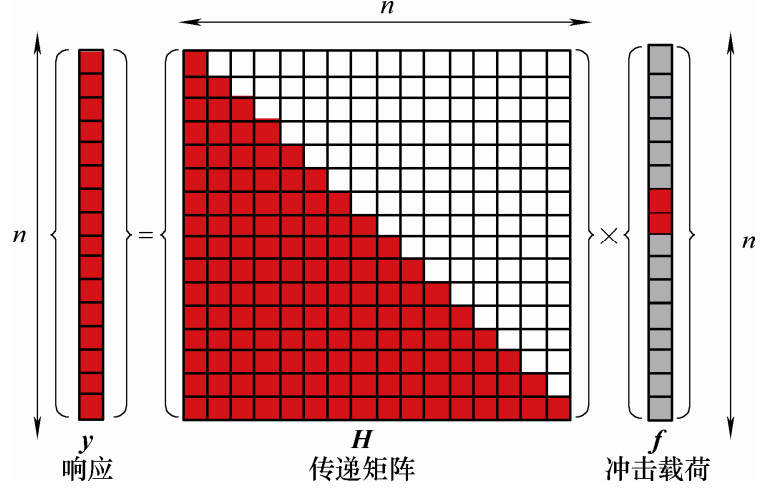

图 1 单源冲击载荷稀疏识别模型

表面上看, 基于 L1 范数的稀疏正则化模型(15) 与基于 L2 范数的 Tikhonov 正则化模型(9)非常相 似。实际上, 由于目标函数(15)不存在显式解, 二 者的求解方式差异很大。L2 范数正则化模型可借助 奇异值分解技术直接求解，L1 正则化模型不存在显 式解而不得不依赖优化算法。基于 L1 范数的稀疏 识别模型是凸的, 可由一般的凸优化求解器求解。 常用的凸优化算法如内点法、投影梯度法、迭代阈 值算法等已广泛应用到信号、图像的稀疏重构中 ${ }^{[15]}$ 。 本文采用改进的内点算法一一原对偶内点法求解冲 击载荷稀疏识别模型, 具体算法可以参考相关文献 [15,17]，不再赘述。

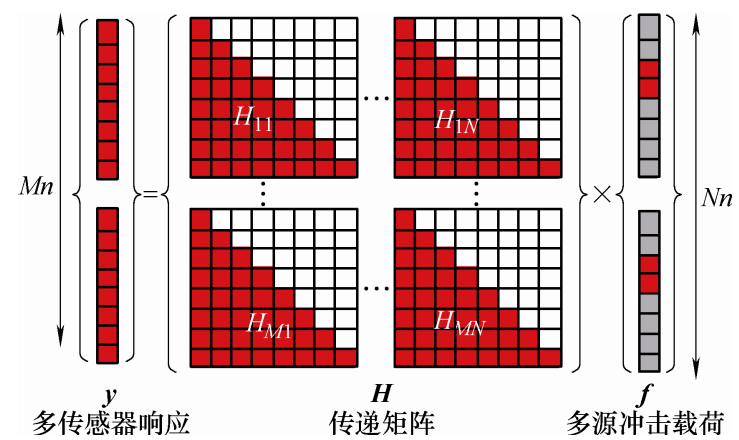

图 2 多源冲击载荷稀疏识别模型

\section{3 试验验证}

将提出的基于 L1 范数的稀疏正则化方法用于 识别作用在悬臂薄板结构的冲击载荷包含单源单次 激励、单源连续激励和多源激励, 并与实测载荷和 基于 L2 范数的 Tikhonov 解作对比, 验证冲击载荷 稀疏识别方法的优势。

\section{1 试验台介绍}

用于验证冲击载荷稀疏识别模型的悬臂薄板结 构如图 3 所示。薄板固定端由若干螺栓紧固, 其它 三边均为自由状态。悬臂板材料是 45 钢, 尺寸参数: 长度 $500 \mathrm{~mm}$ 、宽度 $600 \mathrm{~mm}$ 、厚度 $5 \mathrm{~mm}$ 。薄板表 面均匀划分 25 个网格节点。其中六个位置安放了加 
速度传感器(型号: PCB 333B32, 灵敏度: $100 \mathrm{mV} / \mathrm{g}$ ), 并分别标记为 $\mathrm{R} 1 \sim \mathrm{R} 6$ 。脉冲力锤 (型号: $\mathrm{PCB}$ 086C01, 灵敏度: $12.37 \mathrm{mv} / \mathrm{N}$ )用来对悬臂薄板结构 施加冲击载荷。六路加速度传感器信号和一路载荷 信号由 LMS SCADASIII 数据采集系统以 $2048 \mathrm{~Hz}$ 的频率同步采样。同时, 利用 LMS IMPACT 模块计 算得到任意激励点与六个加速度测点间的频率响应 函数。

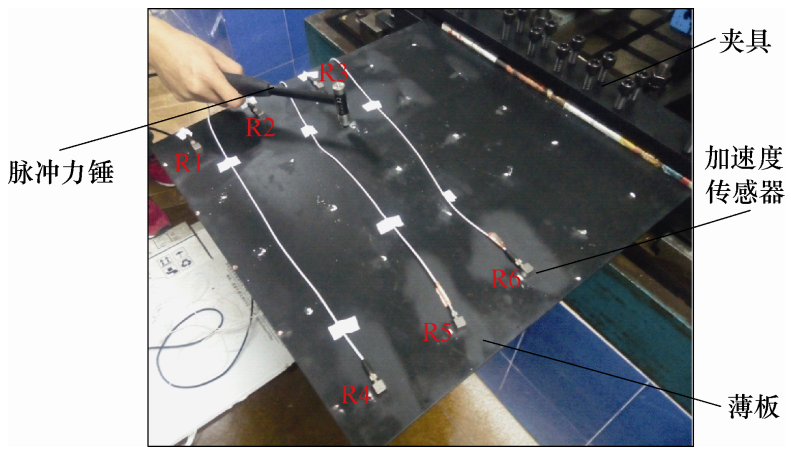

图 3 悬臂薄板结构冲击载荷识别试验

Tikhonov 正则化方法作为一个对比方法亦用来 识别未知冲击载荷, 其中正则化参数由 HANSEN 的 MATLAB 正则化软件包中的 L 曲线准则确定 ${ }^{[4]}$ 。 原对偶内点法的正则化参数为 $\lambda=0.01 \lambda_{\text {max }}$ 。其中, $\lambda_{\text {max }}=\left\|2 \boldsymbol{H}^{\mathrm{T}} \boldsymbol{y}\right\|_{\infty}$, 表示无穷范数。计算环境为一台个 人电脑, 配置为 Intel I5-3450 的 CPU 和 8 GB 的内 存。为了定量评价基于 L1 范数的稀疏识别方法和 基于 L2 范数的 Tikhonov 正则化方法的性能, 将计 算冲击载荷识别结果与实测载荷的相对误差、峰值 相对误差以及相关系数。

\section{2 单源冲击载荷识别}

单源冲击载荷包含单次冲击载荷和两次连击载 荷, 脉冲力锤持续作用在薄板结构某一节点(即 25 个节点中的一个)。注意: 受篇幅限制, 仅以一个激 励点为例说明。图 4 显示了作用在薄板结构的单次 冲击载荷和两次连击载荷在六个测点处的加速度响 应信号。从中可知, 每一个冲击响应信号都是快速 震荡衰减的, 冲击载荷的加速度响应包含前一次加 速度响应信号, 即连续冲击载荷之间的加速度响应 存在 “交叠” 现象, 这里将这种与随机白噪声不同 的干扰称为 “有色噪声”。因此, 用于反演冲击载荷 的加速度响应被有色噪声严重干扰。值得注意的是： 此时, 振动系统不满足载荷识别控制方程(见式(1)) 的零初始条件的假设, 而这在工程现场具有重要意 义, 因为大多机械结构在受到外来冲击载荷作用时 并非处于静止状态, 比如处于运行状态的航空发动 机风扇叶片。

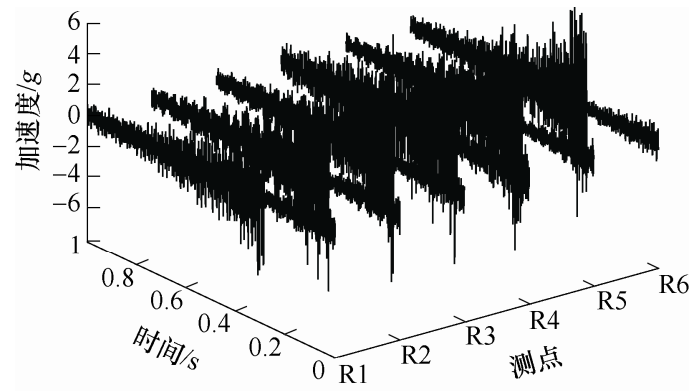

(a) 单次冲击的加速度响应

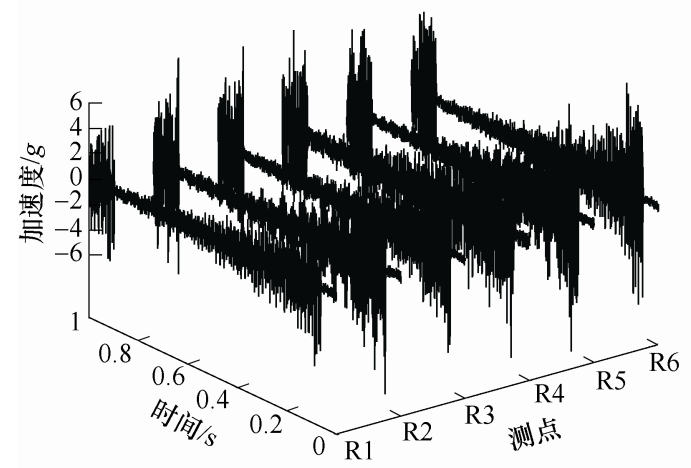

(b) 两次连击的加速度响应

图 4 薄板结构冲击载荷的加速度响应

冲击载荷将由基于 L1 范数的稀疏识别方法和 基于 L2 范数的 Tikhonov 方法分别利用六个测点的 加速度信号反演识别。瀑布图 5 和图 6 显示了两类 正则化方法识别的单次和两次连击冲击载荷。嵌入 力锤顶端的力传感器实测载荷作为真实值(Exact)也 显示在瀑布中的第一个子图中。可知, 实测冲击载 荷在时间区间 $[0,1] \mathrm{s}$ 是一个典型的脉冲信号, 即本 身就具有时域稀疏性。对于单次冲击载荷, 峰值力 在 $t=0.2871 \mathrm{~s}$ 达到最大值。对于两次连击载荷, 峰 值力分别在 $t=0.0620 \mathrm{~s}$ 和 $t=0.8975 \mathrm{~s}$ 达到最大值。 从中可知, 无论是单次还是两次冲击载荷识别, 基 于 L1 范数的稀疏识别方法利用六个测点反演的冲 击载荷均与实测载荷高度吻合; 相反, 基于 L2 范 数的 Tikhonov 方法利用六个测点反演的冲击载荷 均与实测载荷相差较大, 这种差距从图 5 和图 6 中 载荷峰值的比较就显而易见。因此, 稀疏识别方法 可以利用高度污染的测量响应(振动系统初始状态 非静止)精准重构冲击载荷的时间历程, 而当测量噪 声较大时, Tikhonov 难以有效重构冲击载荷。值得 注意的是, 稀疏识别解在冲击力加载区与实测峰值 力高度吻合, 而在非加载区存在一些虚假峰值(小于 $10 \mathrm{~N}$ ), 特别是在有色噪声干扰区间。

表 1 和表 2 具体比较了基于 L1 范数的稀疏正 则化方法和基于 L2 范数的 Tikhonov 方法冲击载荷 识别结果。比较稀疏识别方法单次冲击载荷的相对 误差与两次连击载荷的相对误差, 前者普遍大于后 
者。一个合理的解释是前者测量响应中噪声分布区 间[0, 0.287 1] s 大于后者分布区间 $[0,0.062$ 0] s。考 虑不同测点对稀疏解的影响, 无论是单次冲击还是 两次连击, 测点 $\mathrm{R} 2$ 和 $\mathrm{R} 5$ 反演精度均大于其他测点。 对于冲击载荷识别而言, 峰值精度是评价正则化方 法的一个重要指标。可知, 无论是单次冲击载荷还 是连击载荷的峰值相对误差都在可接受的范围之内 (小于 $6 \%$ )。具体来说, 实测单次冲击载荷峰值力为 $89.65 \mathrm{~N}$ 。以测点 $\mathrm{R} 1$ 反演结果为例, 稀疏正则化解 的峰值力为 $87.47 \mathrm{~N}$, 而 Tikhonov 解的峰值力仅为 $36.57 \mathrm{~N}$ 。实测两次连击载荷的峰值力分别为 103.00 $\mathrm{N}$ 和 $89.37 \mathrm{~N}$ 。同样以测点 $\mathrm{R} 1$ 反演结果为例, 稀疏
正则化解的两个峰值力分别为 $107.70 \mathrm{~N}$ 和 $89.34 \mathrm{~N}$, 而 Tikhonov 解的两个峰值力分别仅为 $55.21 \mathrm{~N}$ 和 $44.64 \mathrm{~N}$ 。在相关性方面，六个测点稀疏解均与实测 载荷有较高的一致性, 相关系数均大于 0.93 ; 而 Tikhonov 解的相关系数均不超过 0.80 。比较两类方 法的计算效率，基于 L1 范数的稀疏识别方法也有 明显的优势。因此，尽管测点位置会对冲击载荷稀 疏识别结果产生影响，但是在实际应用中这种差异 是可接受的。同时, 稀疏识别方法无论选择哪一个 测点总是可以从高度干扰的测量响应中高精度、高 效地重构冲击载荷，而传统的 Tikhonov 正则化方法 对此则无能为力。

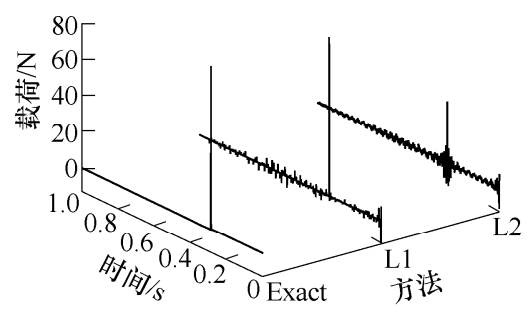

(a) 测点R1反演冲击载荷结果

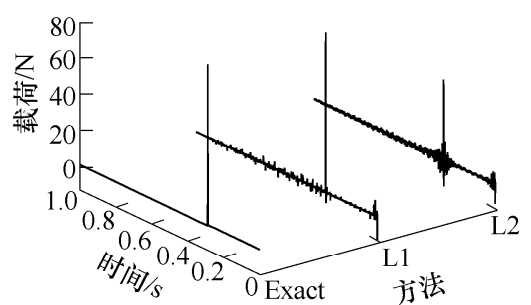

(d) 测点 $\mathrm{R} 4$ 反演冲击载荷结果

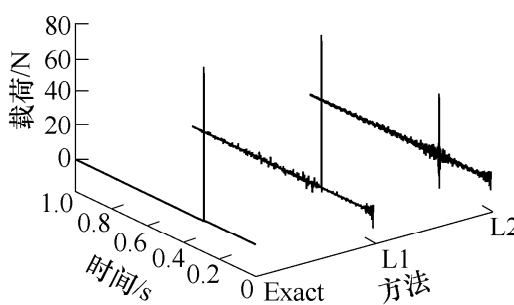

(b) 测点R2反演冲击载荷结果

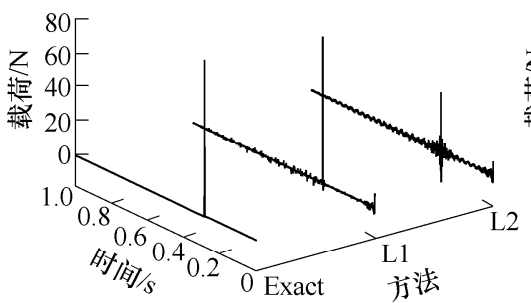

(e) 测点R5反演冲击载荷结果

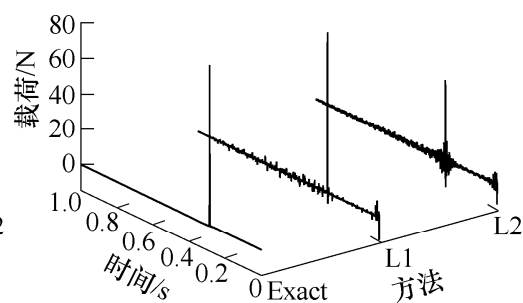

(c) 测点 $\mathrm{R} 3$ 反演冲击载荷结果

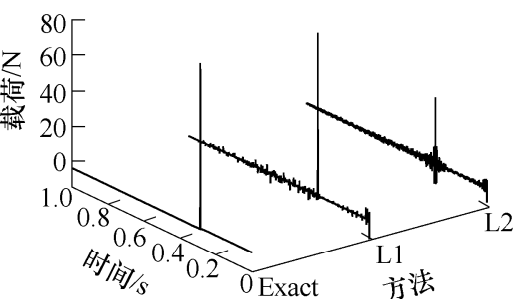

(f) 测点R6反演冲击载荷结果

图 $5 \mathrm{~L} 1$ 和 L2 正则化方法分别采用不同测点识别薄板结构的单源单次冲击载荷

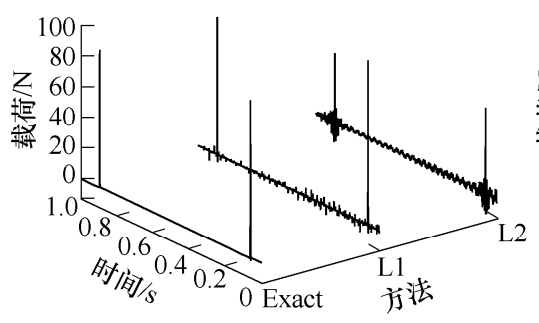

(a) 测点 $\mathrm{R} 1$ 反演冲击载荷结果

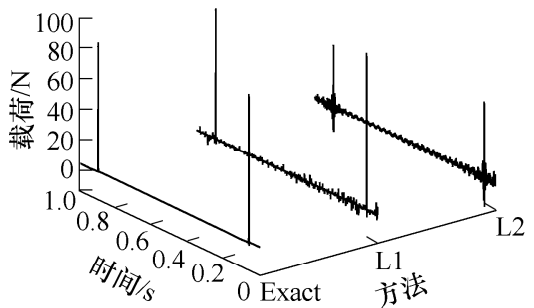

(d) 测点R4反演冲击载荷结果

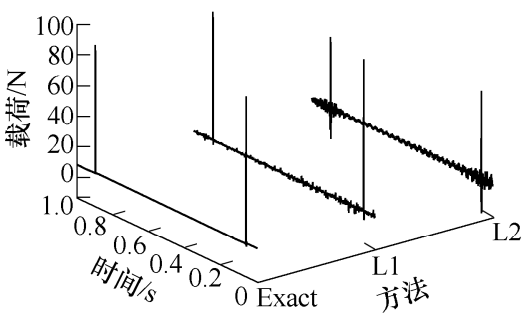

(b) 测点 R2反演冲击载荷结果

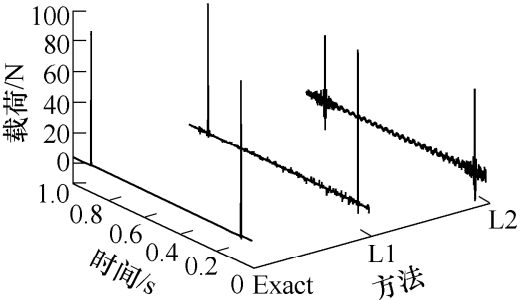

(e) 测点R5反演冲击载荷结果

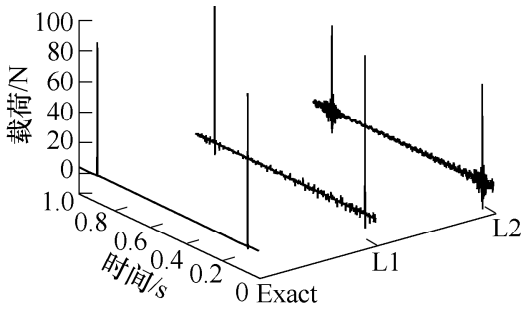

(c) 测点 $\mathrm{R} 3$ 反演冲击载荷结果

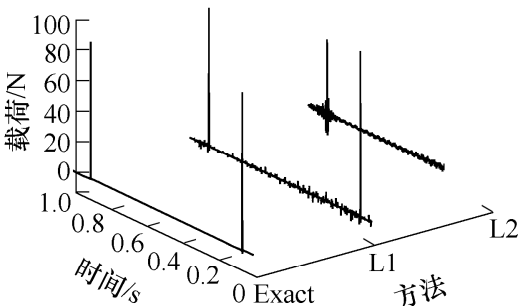

(f) 测点R6反演冲击载荷结果

图 6 L1 和 L2 正则化方法分别采用不同测点识别薄板结构的单源连续冲击载荷 
表 1 针对单源单次冲击载荷, 基于 L1 范数的稀疏识别方法和基于 L2 范数的 Tikhonov 方法识别结果对比

\begin{tabular}{|c|c|c|c|c|c|c|c|}
\hline 方法 & 项目 & $\mathrm{R} 1$ & $\mathrm{R} 2$ & R3 & $\mathrm{R} 4$ & R5 & R6 \\
\hline \multirow{4}{*}{ L1 } & 相对误差(\%) & 28.14 & 23.70 & 29.71 & 37.73 & 19.32 & 26.56 \\
\hline & 峰值相对误差(\%) & 2.44 & 0.48 & 0.03 & 0.60 & 5.92 & 1.09 \\
\hline & 相关系数 & 0.9621 & 0.9734 & 0.9583 & 0.9360 & 0.9827 & 0.9673 \\
\hline & 计算时间/s & 3.36 & 3.46 & 3.87 & 3.94 & 3.91 & 3.03 \\
\hline \multirow{4}{*}{ L2 } & 相对误差(\%) & 78.49 & 80.01 & 76.10 & 80.40 & 82.72 & 76.67 \\
\hline & 峰值相对误差(\%) & 59.21 & 60.08 & 50.34 & 60.43 & 62.60 & 60.21 \\
\hline & 相关系数 & 0.6197 & 0.6028 & 0.6490 & 0.5947 & 0.5629 & 0.6513 \\
\hline & 计算时间/s & 5.14 & 5.10 & 5.50 & 5.09 & 5.11 & 5.17 \\
\hline
\end{tabular}

表 2 针对单源连续冲击载荷, 基于 L1 范数的稀疏识别方法和基于 L2 范数的 Tikhonov 方法识别结果对比

\begin{tabular}{|c|c|c|c|c|c|c|c|}
\hline 方法 & 项目 & $\mathrm{R} 1$ & $\mathrm{R} 2$ & R3 & $\mathrm{R} 4$ & $\mathrm{R} 5$ & R6 \\
\hline \multirow{5}{*}{ L1 } & 相对误差(\%) & 19.22 & 15.60 & 19.46 & 21.08 & 13.80 & 18.25 \\
\hline & 1st 峰值相对误差(\%) & 4.56 & 3.20 & 3.50 & 5.73 & 1.17 & 5.44 \\
\hline & 2nd 峰值相对误差(\%) & 0.03 & 0.23 & 2.58 & 1.84 & 3.75 & 1.49 \\
\hline & 相关系数 & 0.9825 & 0.9890 & 0.9824 & 0.9809 & 0.9914 & 0.9860 \\
\hline & 计算时间 $/ \mathrm{s}$ & 3.15 & 3.71 & 3.64 & 3.62 & 3.70 & 3.51 \\
\hline \multirow{5}{*}{ L2 } & 相对误差(\%) & 68.75 & 67.76 & 65.94 & 5.63 & 73.97 & 64.38 \\
\hline & 1st 峰值相对误差(\%) & 46.41 & 40.31 & 36.66 & 49.16 & 47.16 & 42.02 \\
\hline & 2nd 峰值相对误差(\%) & 50.76 & 45.15 & 36.54 & 52.64 & 51.82 & 45.37 \\
\hline & 相关系数 & 0.7308 & 0.7391 & 0.7525 & 0.7129 & 0.6749 & 0.7735 \\
\hline & 计算时间 $/ \mathrm{s}$ & 5.22 & 5.08 & 5.28 & 5.16 & 5.14 & 5.02 \\
\hline
\end{tabular}

\section{3 多源冲击载荷识别}

基于 L1 范数的稀疏正则化方法利用两个测点 的加速度响应识别作用在薄板结构的多源冲击载 荷, 并与基于 L2 范数的 Tikhonov 正则化方法对比。 为了模拟多源冲击载荷, 脉冲力锤在极短的时间内 先后作用于两个不同节点。此时, 响应矢量 $\boldsymbol{y}$ 由测 点 R5 和 R6 组装而成, 大小为 4 100; 总体观测矩 阵由两个激励点和两个响应点间的四个观测矩阵组 成, 大小为 4 100×4 100。图 7 显示了先后作用在薄 壳结构 3\#和 8\#节点的冲击载荷产生的加速度响应。 可知, $3 \#$ 节点的冲击响应与 $8 \#$ 节点的冲击响应之间 存在严重的 “交叠”, 且冲击响应之前存在有色噪声 干扰, 即薄板结构初始状态非静态。

稀疏识别方法利用测点 R5 和 R6 反演多源冲击 载荷结果如图 8a 所示; Tikhonov 方法利用测点 R5 和 R6 反演得到多源冲击载荷结果如图 $8 \mathrm{~b}$ 所示。为 了便于比较, 实测的冲击载荷也绘制在瀑布图 8 中 (位置 $=0)$ 。注意：实测的两源冲击载荷信号由同一 个力锤作用在不同位置而产生, 即由同一个力传感 器采集。可知基于 L1 范数的稀疏解均与实测冲击 载荷高度吻合, 且在冲击载荷非加载区数值非常小; 相反, Tikhonov 解严重偏离实测载荷, 且在载荷非 加载区存在若干较大峰值力。实测的 $3 \#$ 和 $8 \#$ 节点的 冲击载荷峰值力分别为 $71.65 \mathrm{~N}$ 和 $90.78 \mathrm{~N}$, 作用时 间分别为 $t_{1}=0.4370 \mathrm{~s}$ 和 $t_{2}=0.7168 \mathrm{~s}$ 。稀疏识别方法
求解的作用于 $3 \#$ 和 $8 \#$ 节点冲击载荷的峰值力分别 为 $69.27 \mathrm{~N}$ 和 $92.79 \mathrm{~N}$, 对应的峰值误差分别仅为 $3.25 \%$ 和 $2.16 \%$ 。 Tikhonov 方法识别的作用于 3\#和 8 \#节点冲击载荷的峰值力分别仅为 $40.45 \mathrm{~N}$ 和 40.43 $\mathrm{N}$, 对应的误差高达 43.55\%和 56.61\%。另外，稀疏 识别方法求解该两输入两输出载荷识别反问题的计 算时间为 $22.07 \mathrm{~s}$, 而 Tikhonov 方法耗时高达 $38.06 \mathrm{~s}$ 。

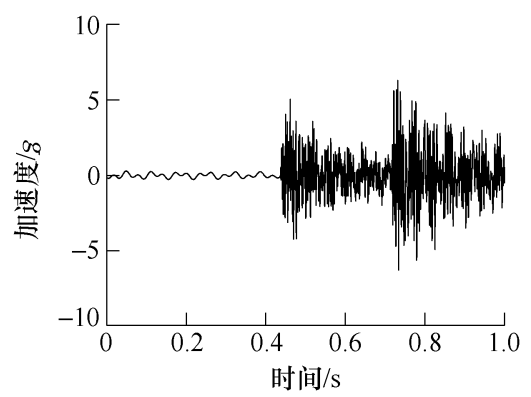

(a) 测点R5的加速度响应

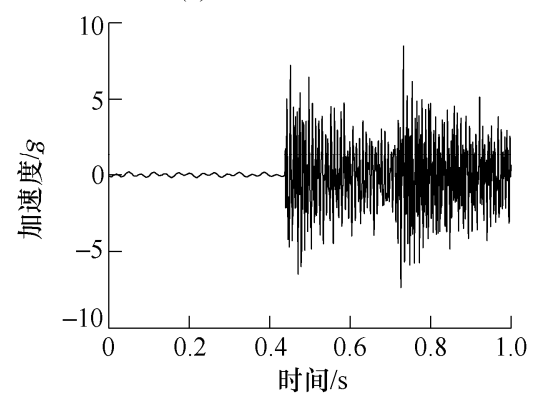

(b) 测点R6的加速度响应

图 7 薄板结构 $3 \#$ 和 $8 \#$ 节点两源冲击载荷的加速度响应 
因此, 与 Tikhonov 方法相比, 稀疏识别方法可以高 精度、高效率地识别多源冲击载荷, 且对有色噪声 不敏感, 即对振动系统初始条件不敏感。

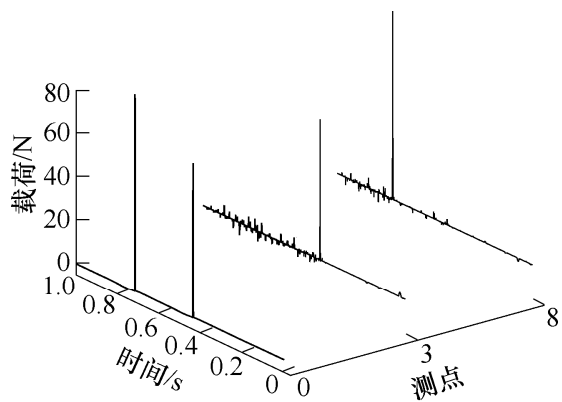

(a) 基于L1范数的稀疏识别结果

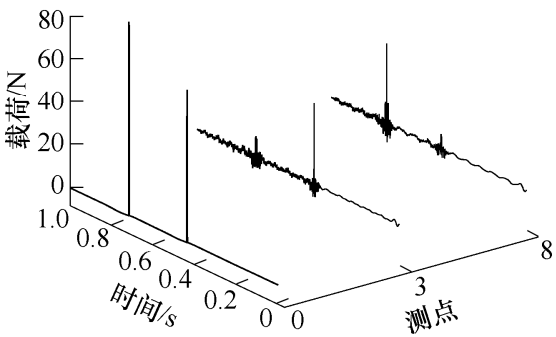

(b) 基于L2范数的Tikhonov识别结果

图 8 薄板结构 3\#和 8\#节点两源冲击载荷的识别结果

\section{4 结论}

（1）从冲击载荷信号的时域稀疏性出发, 提出 了一种新型冲击载荷识别通用模型——稀疏识别正 则化模型, 突破了 L2 范数框架下传统正则化方法 的载荷识别局限性, 极大地抑制了响应噪声在冲击 载荷中的放大, 高精度和高效地解决了冲击载荷识 别难题。其中，构造的基于 L1 范数的稀疏解卷积 模型的通用性体现在可识别具有稀疏特征的各类冲 击载荷和可由众多成熟的凸优化算法求解。

（2）薄板结构单源(单次、多次)和多源冲击载荷 识别试验表明, 冲击载荷的稀疏解在时域内非常稀 疏, 响应噪声被极大地抑制, 相反, 测量噪声在 Tikhonov 解的非加载区被极大地放大; 基于 L1 范 数的稀疏正则化方法均有较高精度的识别结果, 而 经典的 Tikhonov 正则化方法的精度较低; 稀疏识别 方法对振动系统初始条件不敏感, 并不要求振动系 统满足零初始条件的假设, 同时对正则化参数选择 不敏感。值得注意的是, Tikhonov 方法在很多情况 亦可以得到较高精度的识别结果, 然而正则化参数 准则如 $\mathrm{L}$ 曲线并不总能给出最优的参数。

\section{参 考 文 献}

[1] 杨智春, 贾有. 动载荷识别方法的研究进展 [J]. 力学进 展，2015，45: 201502.
YANG Zhichun, JIA You. The identification of dynamic loads[J]. Advances in Mechanics， 2015， 45: 201502.

[2] 卢立勤, 乔百杰, 张兴武, 等. 共轭梯度最小二乘迭代 正则化算法在冲击载荷识别中的应用 $[\mathrm{J}]$. 振动与冲击, 2016, 35(22): 182-189.

LU Liqin, QIAO Baijie, ZHANG Xingwu, et al. Application of conjugate gradient least squares iteration regularization algorithm in impact load identification[J]. Journal of Vibration and Shock. 2016，35(22): 182-189.

[3] QIAO B, ZHANG X, GAO J, et al. Impact-force sparse reconstruction from highly incomplete and inaccurate measurements[J]. Journal of Sound and Vibration, 2016, 376: $72-94$

[4] HANSEN P C. Rank-deficient and discrete ill-posed problems[M]. SIAM, Philadelphia, 1998.

[5] QIAO B, ZHANG X, WANG C, et al. Sparse regularization for force identification using dictionaries[J]. Journal of Sound and Vibration， 2016， 368： 71-86.

[6] DOYLE J F. A wavelet deconvolution method for impact force identification[J]. Experimental Mechanics, 1997, 37(4): 403-408.

[7] WANG L, HAN X, LIU J, et al. A new regularization method and application to dynamic load identification problems[J]. Inverse Problems in Science and Engineering, 2011, 19(6): 765-776.

[8] JACQUELIN E, BENNANI A, HAMELIN P. Force reconstruction : Analysis and regularization of a deconvolution problem[J]. Journal of Sound and Vibration, 2003, 265(1): 81-107.

[9] BOUKRIA Z, PERROTIN P, BENNANI A. Experimental impact force location and identification using inverse problems: Application for a circular plate[J]. International Journal of Mechanics, 2011， 5(1): 48-55.

[10] KALHORI H, YE L, MUSTAPHA S. Inverse estimation of impact force on a composite panel using a single piezoelectric sensor[J]. Journal of Intelligent Material Systems and Structures, 2016, 28(6).

[11] YAN G, ZHOU L. Impact load identification of composite structure using genetic algorithms[J]. Journal of Sound and Vibration, 2009, 319(3): 869-884.

[12] QIAO B, ZHANG X, LUO X, et al. A force identification method using cubic B-spline scaling functions [J]. Journal of Sound and Vibration， 2015，333: 28-44.

[13] QIAO B, CHEN X, XUE X, et al. The application of cubic B-spline collocation method in impact force identification[J]. Mechanical Systems and Signal Processing, 2015, 64-65: 413-427.

[14] QIAO B, CHEN X, LUO X, et al. A novel method for force identification based on the discrete cosine 
transform[J]. ASME Journal of Vibration and Acoustics, 2015, 137(5): 051012.

[15] TROPP J, WRIGHT S J. Computational methods for sparse solution of linear inverse problems[J]. Proceedings of the IEEE，2010，98(6): 948-958.

[16] 张晗, 杜朝辉, 方作为, 等. 基于稀疏分解理论的航空发 动机轴承故障诊断[J]. 机械工程学报, 2015, 51(1): 97-105. ZHANG Han, DU Zhaohui, FANG Zuowei, et al. Sparse decomposition based aero-engine's bearing fault diagnosis[J]. Journal of Mechanical Engineering, 2015, 51(1): 97-105.

[17] QIAO B, ZHANG X, GAO J, et al. Sparse deconvolution for the large-scale ill-posed inverse problem of impact force reconstruction[J]. Mechanical Systems and Signal
Processing, 2017, 83: 93-115.

[18] CHEN S S, DONOHO D L, SAUNDERS M A. Atomic decomposition by basis pursuit[J]. SIAM Journal on Scientific Computing, 1998, 20(1): 33-61.

[19] DONOHO D L. For most large underdetermined systems of linear equations the minimal 11-norm solution is also the sparsest solution[J]. Communications on Pure and Applied Mathematics, 2006, 59(6): 797-829.

作者简介: 乔百杰, 男, 1985 年出生, 博士, 讲师。主要研究方向为载 荷识别、结构动力学反演理论、航空发动机叶片动应变场重构。

E-mail: qiao1224@xjtu.edu.cn

陈雪峰(通信作者), 男, 1975 年出生, 博士, 教授, 博士研究生导师。 主要研究方向为机械装备动态分析与故障诊断。

E-mail: chenxf@xjtu.edu.cn 